\title{
CHAPTER
}

\section{Processing of Transmission Electron Microscopy Images for 16 Single-Particle Analysis of Macromolecular Complexes}

\author{
Zaelle Devaux*, Carlos Oscar S. Sorzano ${ }^{\dagger}$, Slavica Jonic ${ }^{*, 1}$ \\ *IMPMC, UMR 7590, CNRS-Université Pierre and Marie Curie-IRD, Campus Jussieu, \\ Paris Cedex 05, France \\ ${ }^{\dagger}$ Biocomputing Unit, Centro Nacional de Biotecnología-CSIC, \\ Campus de Cantoblanco, Madrid, Spain \\ ${ }^{\prime}$ Corresponding author: slavica.jonic@impmc.jussieu.fr
}

\section{CHAPTER OUTLINE}

1 Purpose

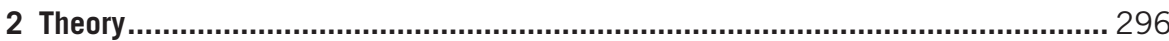

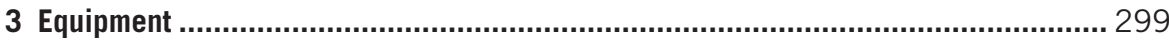

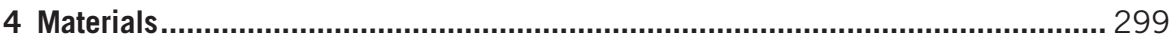

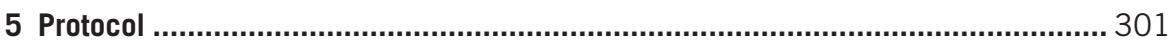

5.1 Step 1-Screen Micrographs and Estimate the CTF Parameters ............... 302

5.2 Step 2-Pick and Screen Particles ............................................... 303

5.3 Step 3-Create Defocus Groups and Prepare the First Reference Volume...................................................... 305

5.4 Step 4-Refine Iteratively the 3D Structure by Projection Matching with CTF Correction ................................................................... 305

\section{Abstract}

Three-dimensional (3D) structure of a wide range of biological macromolecular assemblies can be computed from two-dimensional images collected by transmission electron microscopy. This information integrated with other structural data (e.g., from X-ray crystallography or nuclear magnetic resonance) helps structural biologists understand the function of macromolecular complexes. Single-particle analysis (SPA) is a method used for studies of complexes whose structure and dynamics can be analyzed in isolation. To reconstruct the 3D structure, SPA methods use a large number of images of randomly oriented individual complexes. When the angular 
distribution of single-particle orientations samples Fourier space completely and the population is structurally homogeneous, a resolution of the reconstruction of 0.4-1 $\mathrm{nm}$ can be achieved. Such high resolutions are possible thanks to the high number of images and the correction of the Contrast Transfer Function (CTF) of the microscope. One of the standard SPA approaches is the refinement of a preliminary 3D model using iterative projection matching combined with CTF correction. We describe a protocol for the refinement of a preliminary model using CTF correction by Wiener filtering of volumes from focal series of experimental images. This protocol combines potentially best features of two other protocols proposed in the field.

\section{PURPOSE}

The purpose of this protocol is to compute a three-dimensional structure of a macromolecular complex by single-particle analysis of transmission electron microscopy images.

\section{THEORY}

Three-dimensional (3D) structure of a widerange of biological macromolecular assemblies can be computed from two-dimensional (2D) images collected by transmission electron microscopy. This information integrated with other structural data (e.g. from $\mathrm{X}$-ray crystallography) helps structural biologists understand the function of macromolecular complexes. Single-particle analysis (SPA) is a method used for studies of macromolecular assemblies whose structure and dynamics can be analyzed in isolation (e.g. proteins, ribosomes, viruses) (Frank 2006; Jonic et al., 2008; jonic and Venien-Bryan, 2009). It is complementary to nuclear magnetic resonance since it allows computing the structure of large assemblies (diameter of $10-30 \mathrm{~nm}$ ). It is also complementary to X-ray crystallography since it allows studying noncrystalline matter.

To reconstruct the 3D structure, SPA methods use a large number of images of randomly oriented individual molecules (Fig. 1). In practice, the analysis requires images of thousands of individual molecules of the same protein captured in a unique conformation taken in random orientation. Note that we do not treat, in this protocol, the case of the samples with structural heterogeneity (for a review on this topic, see Leschziner $\&$ Nogales, 2007). When the angular distribution of single-particle orientations samples Fourier space completely and the population is structurally homogeneous, standard image processing strategies allow computing an average structure at resolution of 0.4-1 nm (Connell et al., 2007; Cottevieille et al., 2008; Ludtke et al., 2008; VenienBryan et al., 2009; Zhang et al., 2008). Such high resolutions are possible thanks to a high number of images used for $3 \mathrm{D}$ reconstruction and the correction of the contrast transfer function (CTF) of the microscope. The CTF is expressed in reciprocal space and its equivalent in real space is termed point spread function as it describes how the image of a single point is spread into a diffused spot because of the imperfections of the electron microscope (e.g. spherical and chromatic aberrations, instabilities of magnetic lenses, instabilities of electron acceleration, additive noise, drift or charging). 


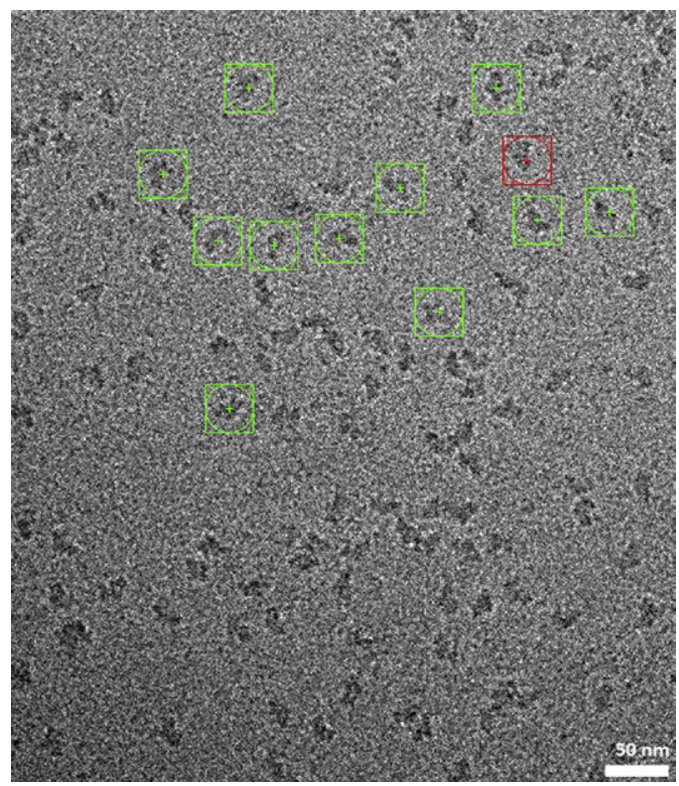

FIGURE 1

Area of a micrograph containing hundreds of single particles (squares) in random and unknown orientations. See the color plate.

One of the standard SPA methods is the refinement of a preliminary 3D model using iterative projection matching combined with CTF correction. An already solved structure from the same family of macromolecular complexes can be downloaded from the PDB database (http://www.ebi.ac.uk/pdbe/) or the EMDB database (http://www.ebi.ac.uk/pdbe/emdb/) and used as the preliminary model, after an appropriate low-pass filtering to remove fine structural details, and thus prevent biasing the outcome by the high-resolution features in the initial reference (Fig. 2). If such structure is not available, other techniques can help obtain the preliminary model (e.g. random conical tilt series (Radermacher, 1988), common lines (Penczek, Zhu, \& Frank, 1996)). Here, we describe a protocol for the refinement of a preliminary PDB or EMDB model using CTF correction by Wiener filtering of volumes from focal series of experimental images (Penczek, Zhu, Schröder, \& Frank, 1997) thanks to which we obtained several low-symmetry structures at subnanometer resolution (Cottevieille et al., 2008; Venien-Bryan et al., 2009). This protocol is inspired by two from several protocols proposed in the field (Scheres, Nunez-Ramirez, Sorzano, Carazo, \& Marabini, 2008; Shaikh et al., 2008) and aims at combining potentially best features of both.

The experimental images to be used for reconstruction are first screened on two levels. On the micrograph level, the screening is done to detect and reject micrographs with important information loss (e.g. due to thermal drift effects) or high 

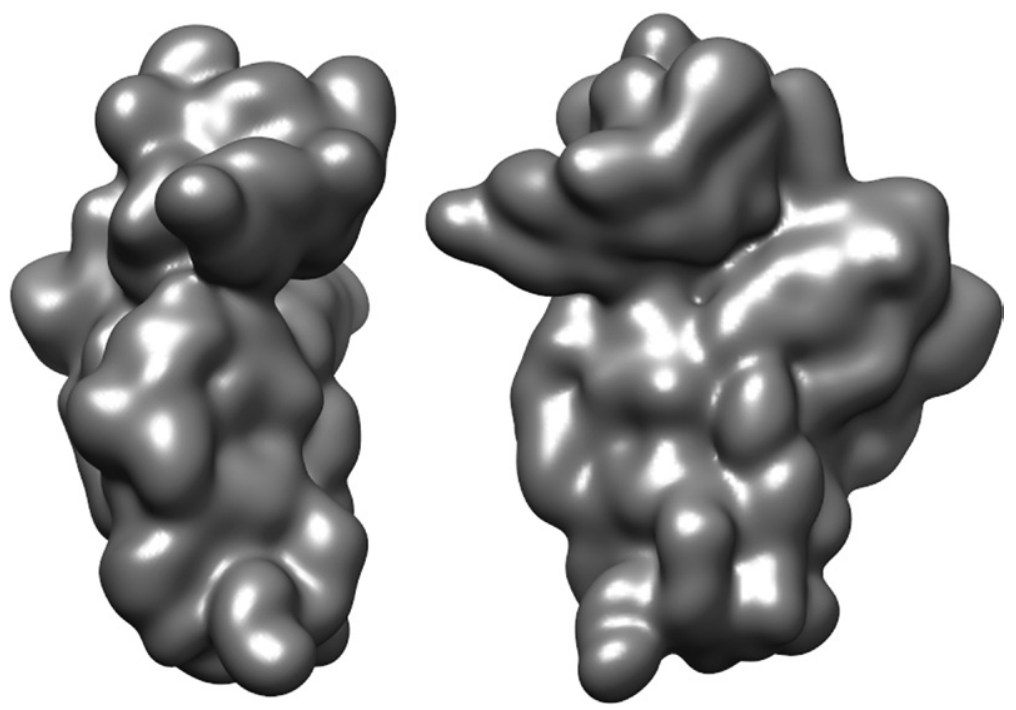

FIGURE 2

Two views of the structure of the ribosomal subunit $30 \mathrm{~S}$ of $T$. thermophilus downloaded from the Protein Data Bank (PDB code: 1j5e), low-pass filtered, and used as the preliminary model for 3D reconstruction of the subunit 30S of $P$. abyssi.

astigmatism in case of Wiener filtering of volumes from focal series (Jonic, Sorzano, Cottevieille, Larquet, \& Boisset, 2007) (Fig. 3). At the same time, the CTF parameters are estimated for each micrograph (Sorzano et al., 2007) (Fig. 4). On the level of isolated single-particle images, the classification is done to detect and reject the images containing the structures inconsistent with the structure of the remaining particles (Sorzano et al., 2010) (Fig. 5).

The kept single particles are classified into groups containing images with similar defocus values. For each defocus group, the 3D defocus-group CTF is applied on the 3D reference. The CTF affected volume is then projected using known projection directions (known angular sampling step) to compute a library of 2D reference projections with known orientations. The experimental single-particle images are then correlated with the reference projections (projection matching of the 3D reference with experimental images) to compute roughly the orientation of the experimental images (Jonic et al., 2005; Sorzano et al., 2004). The oriented images in each defocus group are used for the $3 \mathrm{D}$ reconstruction of the corresponding defocusgroup volume and the volumes are merged using a Wiener filter computed for the defocus-groups CTF parameters (Penczek et al., 1997). Until the resolution of the reconstructed structure becomes stable (Fig. 6), this merged CTF-corrected volume is used as the refined 3D reference for the next iteration, in which the projection matching is performed with the reference projections computed for a smaller angular sampling step. 

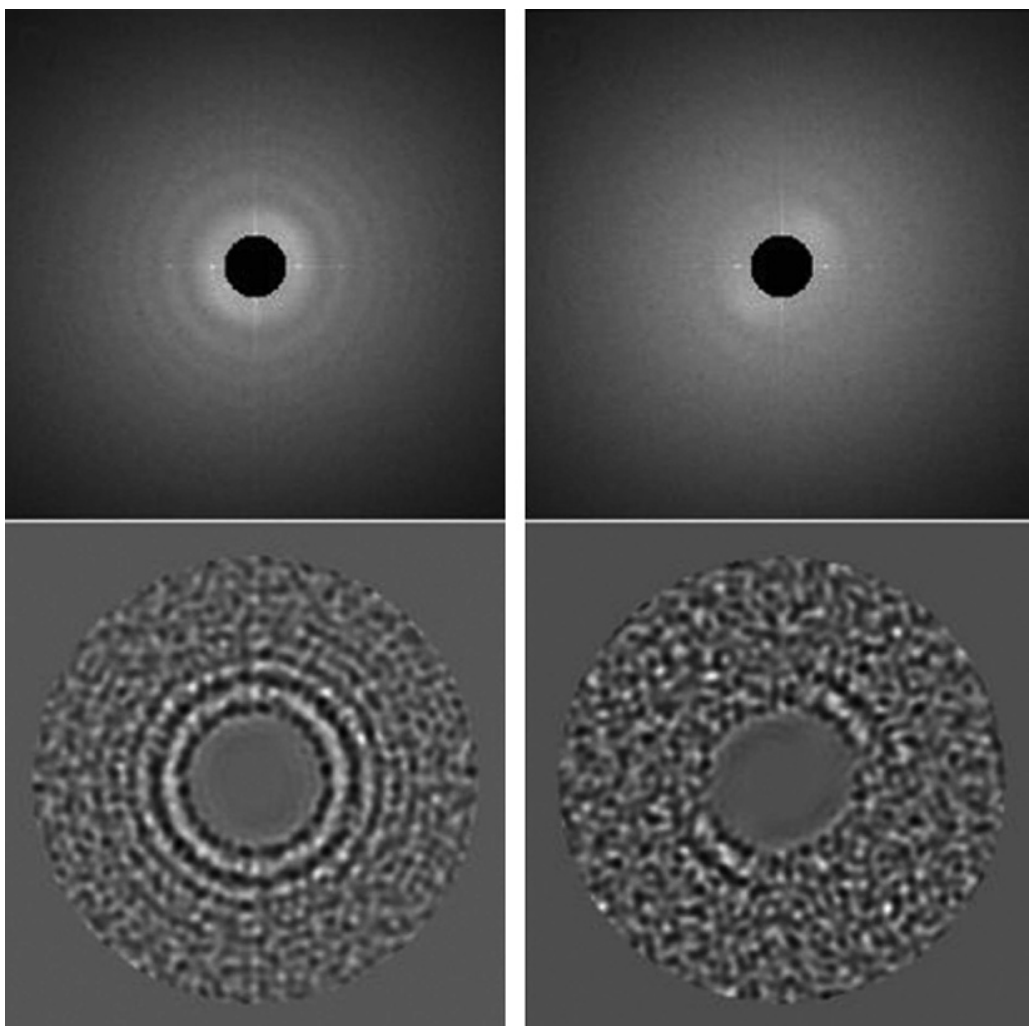

\section{FIGURE 3}

Screening of micrographs to detect thermal drift effects and astigmatism. Top panels: power spectral density of experimental images, with masked very low frequencies to improve the visibility of contrast transfer function rings. Bottom panels: band-pass filtered power spectral densities to improve the rings visibility, with masked very low and very high frequencies. Left panels: rings circularly symmetric (absence of astigmatism). Right panels: rings visible only in one direction (thermal drift).

\section{EQUIPMENT}

Desktop PC with multiple processing cores or a cluster.

\section{MATERIALS}

Linux operating system

SPA software Xmipp (http://xmipp.cnb.csic.es) or Spider (http://www.wadsworth. org/spider_doc)

$2 \mathrm{D}$ visualization software (e.g. provided by SPA package)

$3 \mathrm{D}$ visualization software (e.g. Chimera (http://www.cgl.ucsf.edu/chimera)) 


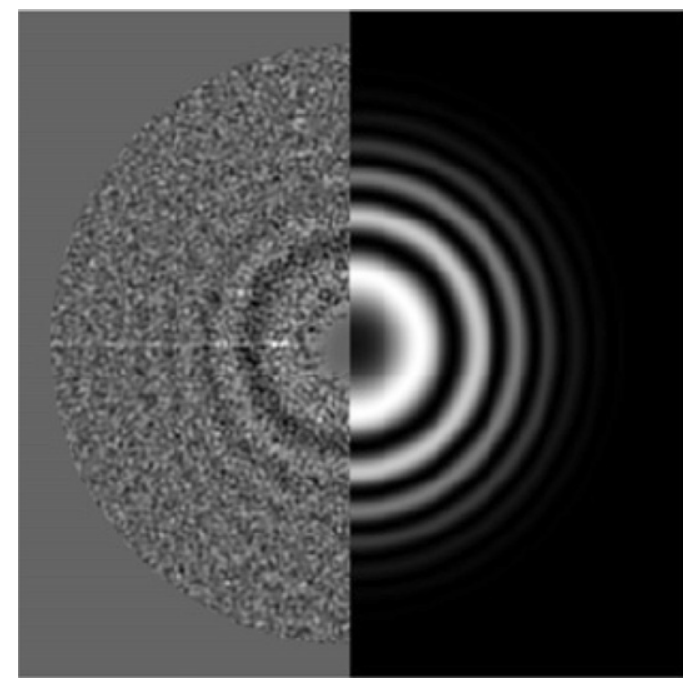

\section{FIGURE 4}

Combined image composed of the left half of the band-pass filtered power spectral density and the right half of the estimated two-dimensional contrast transfer function to check the accuracy of the estimation of the contrast transfer function.

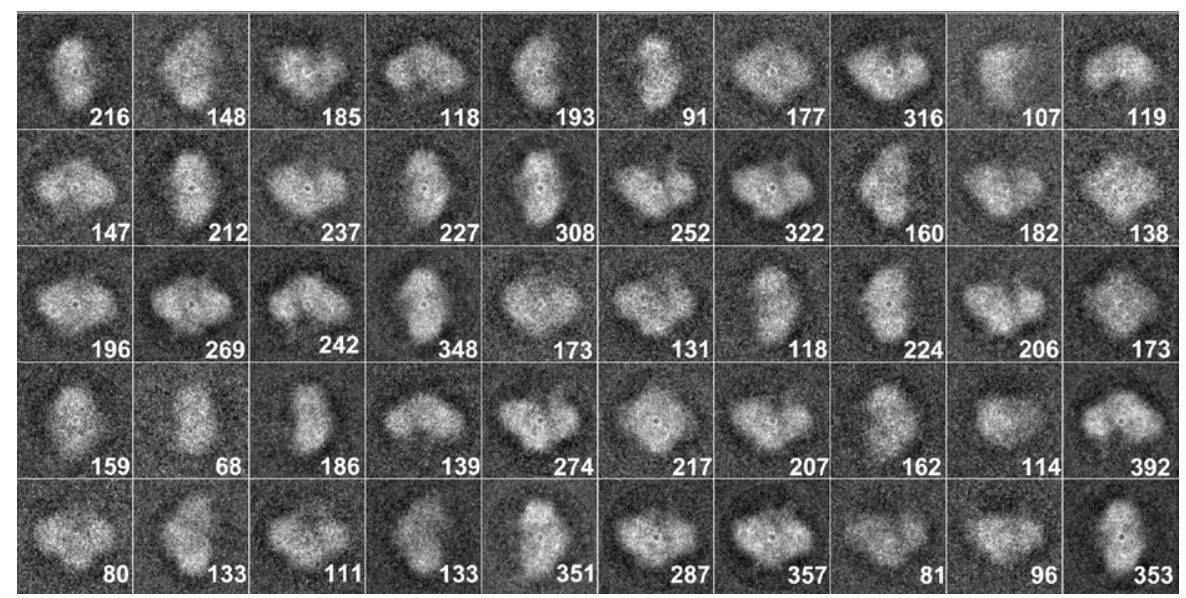

\section{FIGURE 5}

Screening of single particle images to detect heterogeneity. Classification of around 10,000 single particles in 50 classes, considered as homogeneous. Class averages with the number of particles indicated for each class. 

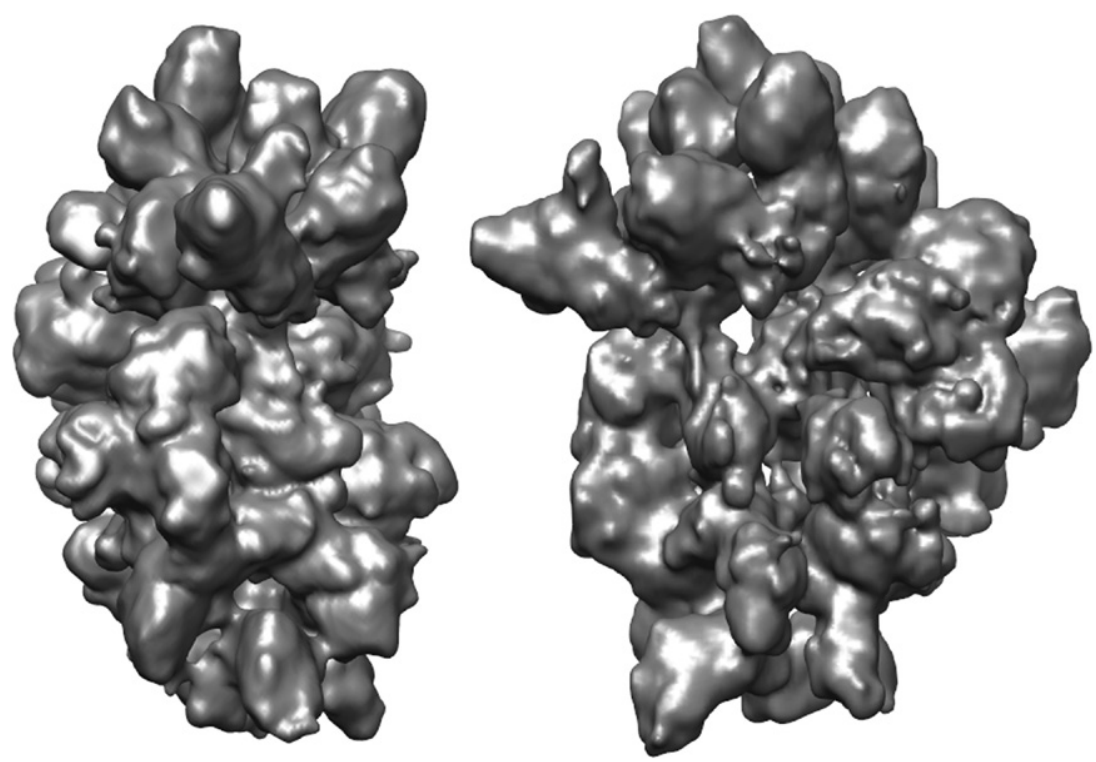

\section{FIGURE 6}

Two views of the structure of the ribosomal subunit $30 S$ of $P$. abyssi obtained at around $1 \mathrm{~nm}$ resolution (corresponding to the Fourier shell correlation of 0.5 ) by refinement of the $30 \mathrm{~S}$ structure of $T$. thermophilus (Fig. 2). Iterative projection matching of 9523 single-particle images was combined with contrast transfer function correction by Wiener filtering of five volumes from focal series, with the computed defocus in the range from -0.6 to $-2.7 \mu \mathrm{m}$.

\section{PROTOCOL}

\begin{tabular}{cl|l} 
Duration & & Time \\
& Preparation & 30 min \\
Protocol & $7-8$ days \\
\hline
\end{tabular}

Prepa- $\quad$ Obtain a preliminary 3D model of the studied complex by searching and ration downloading from the PDB or EMDB databases an available structure of a similar complex.

Caution In case the PDB or EMDB databases do not contain an appropriate structure, use the image acquisition and analysis techniques specially designed for obtaining the first 3D model such as random conical tilt series or common lines techniques (out of scope of this article). 


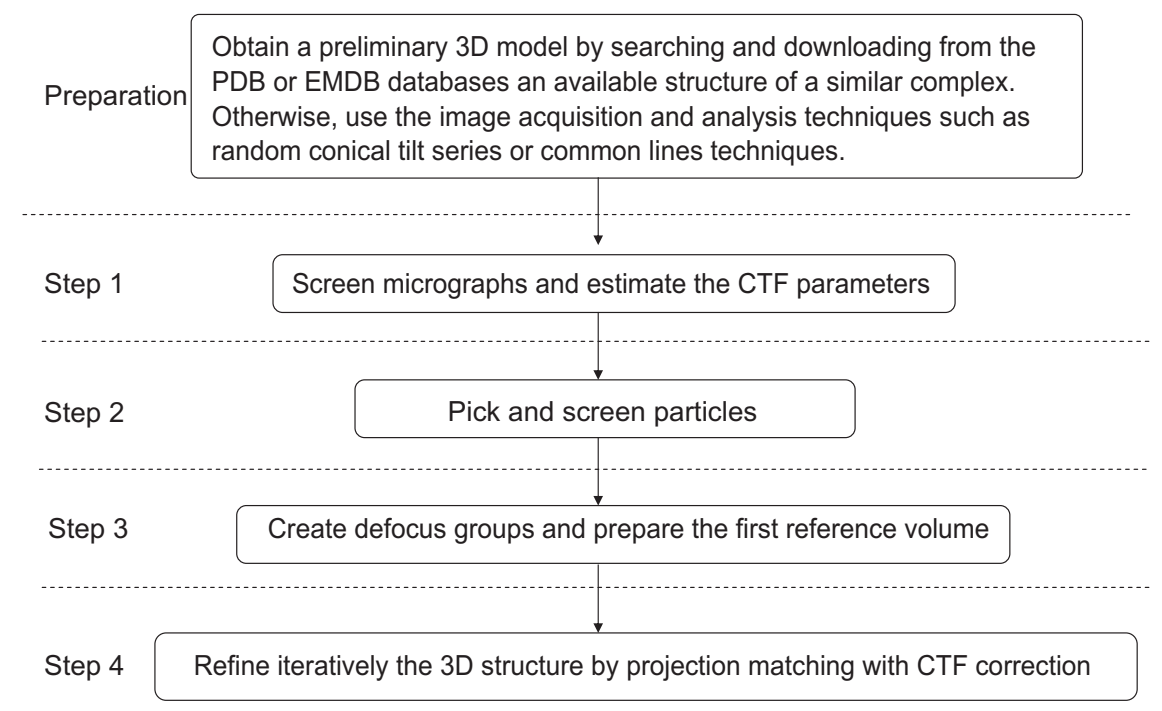

FIGURE 7

Flowchart of the complete protocol, including preparation.

Tip Duration reported here includes both computing time and time for various intermediate results analyses. Computing time can be reduced with a higher number of processing cores. Duration reported here is for the use of 32 cores at maximum, intermediate resolution of 3D reconstruction $(\sim 1.5 \mathrm{~nm})$, intermediate-size data set (100 micrographs with a total of about 10,000 particles and the particle size of $128 \times 128$ pixels), and particles of asymmetric form. Required time would be shorter for highly symmetric structures since their reconstruction requires less data to be analyzed for the same target resolution (e.g. icosahedral viruses). Larger the data size, longer the duration but better the resolution of the reconstruction.

See Fig. 7 for the flowchart of the complete protocol.

\subsection{Step 1-Screen Micrographs and Estimate the CTF Parameters}

Overview Screen micrographs to detect and reject micrographs with important information loss (e.g. due to thermal drift effects) and high astigmatism, and estimate the CTF for kept micrographs.

Duration About $3 \mathrm{~h}$.

1.1 Compute 2D power spectrum density (PSD) for each micrograph.

1.2 Compute band-pass filtered PSDs to improve the visibility of the CTF-related rings.

1.3 Detect the PSDs with the CTF-related rings with significant circular asymmetry.

$1.4 \quad$ Remove micrographs giving anisotropic PSDs.

1.5 Compute the CTF parameters for kept micrographs after Step 1.4.

$1.6 \quad$ Visualize a combined image composed of the left half of the band-pass filtered PSD and the right half of the estimated two-dimensional CTF (Fig. 4). 
1.7 Compare the left and right sides of the combined image in Step 1.6 to check the accuracy of the CTF estimation.

1.8 For the micrographs with inaccurate CTF estimation, recompute the CTF by local refinement of given values for the defocus parameters and repeat Steps 1.6 and 1.7 until a successful CTF estimation.

$1.9 \quad$ Keep only the micrographs giving good correspondence between the left and right sides in the combined image in Step 1.6 and keep the corresponding estimated CTF parameters.

$1.10 \quad$ Flip phase of micrographs using computed CTF parameters.

Caution Micrographs can be slightly tilted, which results in different CTF parameters over different areas of the same micrograph.

Tip To avoid mixing particles with very different defocus values, split each micrograph into areas and perform Steps 1.1-1.10 locally (on micrograph's areas instead of analyzing entire micrographs).

See Fig. 8 for the flowchart of Step 1.

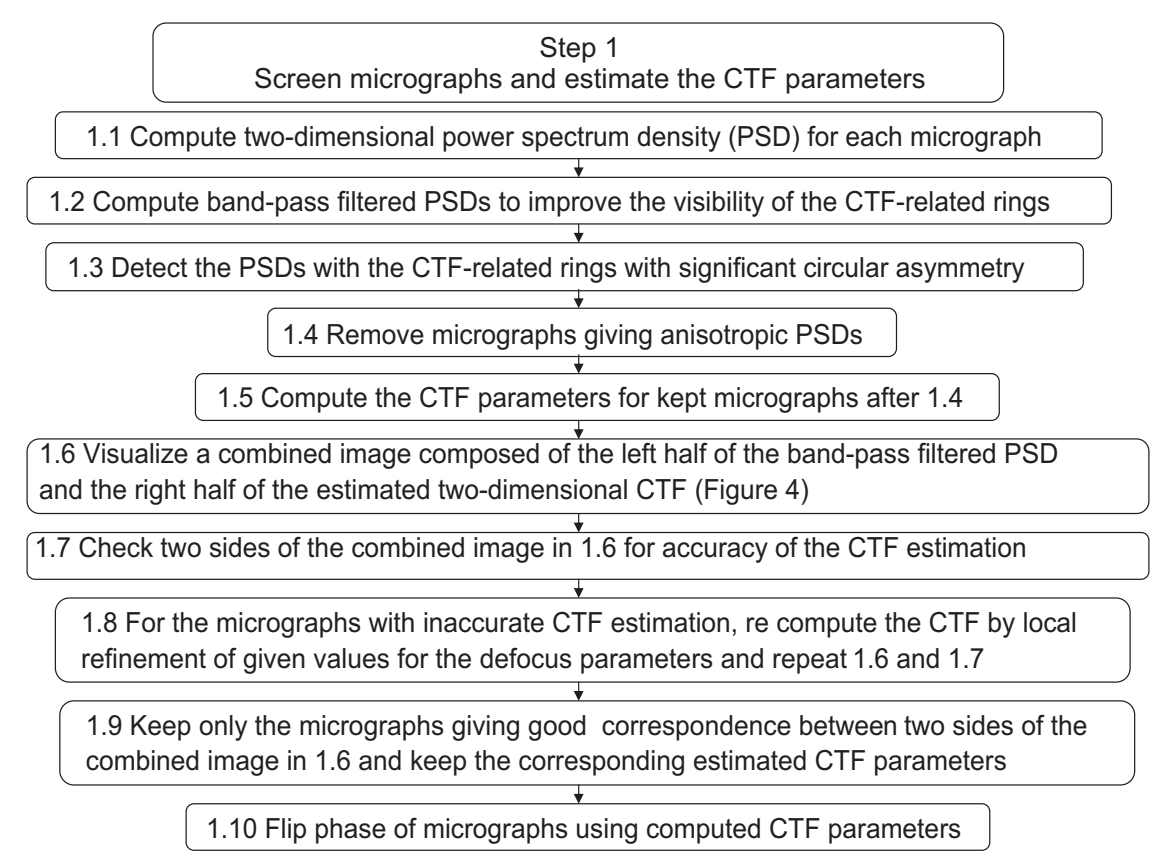

FIGURE 8

Flowchart of Step 1.

\subsection{Step 2-Pick and Screen Particles}

Overview

Isolate particles from micrographs in separate images and detect and keep only structurally homogeneous set of particles.

Duration $\quad$ About 4 days.

2.1 Pick several particles using different box sizes (in pixels) and choose the size of the box that can contain the entire particle. 
2.2 Pick particles semiautomatically using the box size selected in Step 2.1 and store the picked box coordinates.

2.3 Visualize and correct the results of the automatic picking by detecting and rejecting the boxes not containing particles and by picking the missed particles.

2.4 Extract the particles from micrographs using the stored box coordinates.

2.5 Normalize the particles so that the mean and the standard deviation are constant over the series.

2.6 Invert the contrast in case of "black" particles on "white" background.

2.7 Perform classifications of picked particles with different number of classes.

2.8 Visualize the class averages and the individual particles in each class.

2.9 Reject the classes inconsistent with the rest of the classes (produce the final set of particles to be used in the next step).

Tip You can pick the particles using any of the software packages specialized for SPA since each of them contains an interactive graphical interface for semiautomatic particle boxing and inspection of boxed particles.

See Fig. 9 for the flowchart of Step 2.

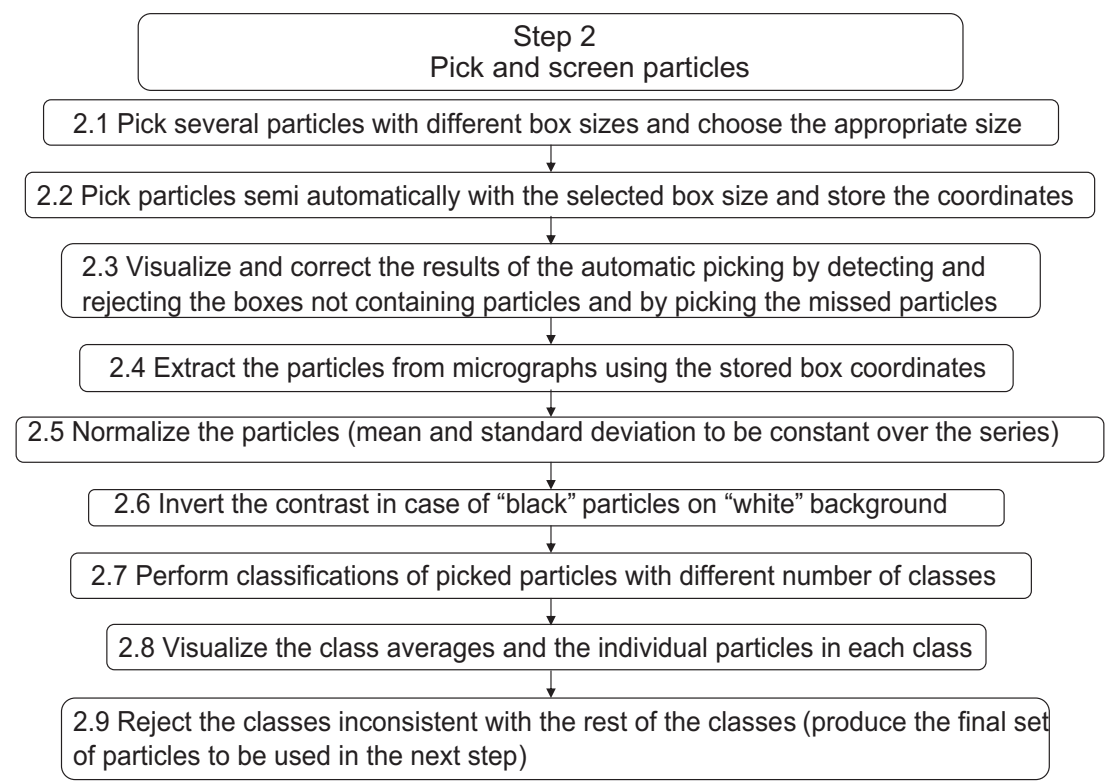

\section{FIGURE 9}

Flowchart of Step 2. 


\subsection{Step 3-Create Defocus Groups and Prepare the First Reference Volume}

Overview $\quad$ Create groups of particles with similar defocus values (defocus groups) and process the available preliminary 3D model to adapt it to the scale of the extracted particle images and remove as much as possible the structural details.

Duration

About $3 \mathrm{~h}$

3.1

Split particles into defocus groups for several given values of the maximum defocus difference in each group.

3.6 Check the number of particles per defocus group and select the split results corresponding to the maximum defocus difference producing the majority of groups with a high number of particles per group (e.g. at least 1000 particles in case of asymmetric structures).

Check the number of particles per defocus group in the split results selected in Step 3.2 and either remove the groups with small number of particles or merge small groups into larger groups, so that all defocus groups have comparable number of particles.

4 Compute the volumetric CTF using the mean defocus value for each defocus group (group CTFs).

Build the reference volume from the preliminary 3D model by rescaling the corresponding density volume, so that its pixel size is that of the particle image and the volume dimension is $N^{3}$ for the particle image size of $N^{2}$ pixels.

Caution Reference-based procedures are sensitive to reference bias.

Tip To avoid biasing of the output with the high-resolution features of the initial volume, low-pass filter the volume in Step 3.6 as much as possible (0.3-0.6 $\mathrm{nm}$ resolution may be required as the cutoff). In addition, it is a good practice to perform independent reconstructions using two (or more) different initial volumes.

See Fig. 10 for the flowchart of Step 3.

\subsection{Step 4-Refine Iteratively the 3D Structure by Projection Matching with CTF Correction}

Overview

Duration

4.1
Images are processed by defocus groups. The projection matching is done in each defocus group with the group CTF applied on the 3D reference. The oriented images in each defocus group are then used to compute one 3D volume per group. The 3D CTF correction is done by merging the volumes from different groups by Wiener filtering and the merged volume is used as the 3D reference for the next iteration.

About 3 days.

For each defocus group, apply the group CTF on the reference volume. 


Step 3
Create defocus groups and prepare the first reference volume

3.1 Split particles into defocus groups for several given values of the maximum defocus difference in each group

3.2 Check the number of particles per defocus group and select the split results producing the majority of groups with a high number of particles per group (e.g. at least 1000 particles in case of asymmetric structures)

3.3 Check the number of particles per defocus group in the split results selected in 3.2 and either remove the groups with small number of particles or merge small groups into larger groups so that all groups have comparable number of particles

3.4 Compute the volumetric CTF using the mean defocus value for each defocus group (group CTFs)

3.5 Build the reference volume from the preliminary 3D model by rescaling the corresponding density volume so that its pixel size is that of the particle image and the volume dimension is $\mathrm{N}^{3}$ for the particle image size of $\mathrm{N}^{2}$ pixels.

3.6 Low-pass filter the volume as much as possible

\section{FIGURE 10}

Flowchart of Step 3.

4.2
For each defocus group, project the obtained group volume using known projection directions to compute a gallery of reference projections.

For each defocus group, compute the particle orientation and shift by matching experimental images with the reference projections.

For each defocus group, perform a 2D realignment of images assigned to each reference projection direction to remove model bias from the refinement procedure.

For each defocus group, compute a 3D reconstruction with the aligned images.

Compute the iteration volume by Wiener filtering of the series of group volumes.

Estimate the resolution limit of the iteration volume by Fourier Shell Correlation of two "half"-volumes (reconstructed from two randomly selected halves of the image series and masked to remove noise around the structure).

Low-pass filter the iteration volume at the estimated resolution (or a little less to avoid overfitting) and mask it to remove noise around the structure. 
4.9

Caution

Tip
Repeat the Steps 4.1-4.8 using the latest iteration volume as the reference volume and reducing the angular step for reference projections computation, until the resolution does not improve anymore.

Mask should be a binary mask with smooth edges (low-pass filter the edges of a binary mask). The mask should not be too small (the mask and structure edges should not be too close to each other).

Start by performing several iterations of the volume refinement without modifying the iteration volume (skip the Steps 4.7 and 4.8). Only after obtaining a volume with significantly reduced noise, create a binary mask that corresponds to the shape of the obtained structure (using several iterations of a combined thresholding and low-pass filtering until the mask of a suitable size is obtained). Then, low-pass filter the edges of the mask and continue the refinement without skipping the Steps 4.7 and 4.8 .

See Fig. 11 for the flowchart of Step 4.

Step 4
Refine iteratively the 3D structure by projection matching with CTF correction

4.1 For each defocus group, apply the group CTF on the reference volume

4.2 For each defocus group, project the obtained group volume using known projection directions to compute a gallery of reference projections

4.3 For each defocus group, compute the particle orientation and shift by matching experimental images with the reference projections

4.4 For each defocus group, perform a 2D re alignment of images assigned to each reference projection direction to remove model bias from the refinement procedure

4.5 For each defocus group, compute a 3D reconstruction with the aligned images

4.6 Compute the iteration volume by Wiener filtering of the series of group volumes

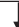

4.7 Estimate the volume resolution by Fourier Shell Correlation of two masked "half"-volumes

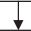

4.8 Low-pass filter the iteration volume at the estimated resolution (or a little less to avoid overfitting) and mask it to remove noise around the structure

4.9 Repeat the steps 4.1 to 4.8 using the latest iteration volume as the reference volume and reducing the angular step for reference projections computation, until the resolution does not improve anymore

\section{FIGURE 11}

Flowchart of Step 4. 


\section{Keywords}

\begin{tabular}{|c|c|c|c|}
\hline Keyword Class & Keyword & Rank & Snippet \\
\hline \multirow{5}{*}{$\begin{array}{l}\text { Methods } \\
\text { List the methods used to } \\
\text { carry out this protocol (i.e. } \\
\text { for each step). }\end{array}$} & $\begin{array}{l}\text { 1. Image } \\
\text { classification }\end{array}$ & & $\begin{array}{l}\text { Used to select structurally } \\
\text { homogeneous set of images }\end{array}$ \\
\hline & 2. Image alignment & & $\begin{array}{l}\text { Used to compute orientation } \\
\text { and translation of images } \\
\text { with respect to a reference }\end{array}$ \\
\hline & $\begin{array}{l}\text { 3. Three- } \\
\text { dimensional (3D) } \\
\text { reconstruction }\end{array}$ & & $\begin{array}{l}\text { Used to compute 3D } \\
\text { structure from 2D images }\end{array}$ \\
\hline & 4. Wiener filter & & $\begin{array}{l}\text { Used to correct contrast } \\
\text { transfer function of the } \\
\text { microscope }\end{array}$ \\
\hline & $\begin{array}{l}\text { 5. Single particle } \\
\text { analysis }\end{array}$ & & $\begin{array}{l}\text { Used to compute an average } \\
\text { structure of a complex from a } \\
\text { high number of individual } \\
\text { particles images }\end{array}$ \\
\hline \multirow{5}{*}{$\begin{array}{l}\text { Process } \\
\text { List the biological } \\
\text { process(es) addressed in } \\
\text { this protocol. }\end{array}$} & 1 & & \\
\hline & 2 & & \\
\hline & 3 & & \\
\hline & 4 & & \\
\hline & 5 & & \\
\hline \multirow{5}{*}{$\begin{array}{l}\text { Organisms } \\
\text { List the primary organism } \\
\text { used in this protocol. List } \\
\text { any other applicable } \\
\text { organisms. }\end{array}$} & 1 & & \\
\hline & 2 & & \\
\hline & 3 & & \\
\hline & 4 & & \\
\hline & 5 & & \\
\hline \multirow{5}{*}{$\begin{array}{l}\text { Pathways } \\
\text { List any signaling, } \\
\text { regulatory, or metabolic } \\
\text { pathways addressed in } \\
\text { this protocol. }\end{array}$} & 1 & & \\
\hline & 2 & & \\
\hline & 3 & & \\
\hline & 4 & & \\
\hline & 5 & & \\
\hline \multirow{5}{*}{$\begin{array}{l}\text { Molecule roles } \\
\text { List any cellular or } \\
\text { molecular roles } \\
\text { addressed in this } \\
\text { protocol. }\end{array}$} & 1 & & \\
\hline & 2 & & \\
\hline & 3 & & \\
\hline & 4 & & \\
\hline & 5 & & \\
\hline \multirow{5}{*}{$\begin{array}{l}\text { Molecule functions } \\
\text { List any cellular or } \\
\text { molecular functions or } \\
\text { activities addressed in } \\
\text { this protocol. }\end{array}$} & 1 & & \\
\hline & 2 & & \\
\hline & 3 & & \\
\hline & 4 & & \\
\hline & 5 & & \\
\hline \multirow{5}{*}{$\begin{array}{l}\text { Phenotype } \\
\text { List any developmental or } \\
\text { functional phenotypes } \\
\text { addressed in this protocol } \\
\text { (organismal or cellular } \\
\text { level). }\end{array}$} & 1 & & \\
\hline & 2 & & \\
\hline & 3 & & \\
\hline & 4 & & \\
\hline & 5 & & \\
\hline
\end{tabular}




\begin{tabular}{|c|c|c|c|}
\hline Keyword Class & Keyword & Rank & Snippet \\
\hline \multirow{5}{*}{$\begin{array}{l}\text { Anatomy } \\
\text { List any gross anatomical } \\
\text { structures, cellular } \\
\text { structures, organelles, or } \\
\text { macromolecular com- } \\
\text { plexes pertinent to this } \\
\text { protocol. }\end{array}$} & 1 & & \\
\hline & 2 & & \\
\hline & 3 & & \\
\hline & 4 & & \\
\hline & 5 & & \\
\hline \multirow{5}{*}{$\begin{array}{l}\text { Diseases } \\
\text { List any diseases or } \\
\text { disease processes } \\
\text { addressed in this } \\
\text { protocol. }\end{array}$} & 1 & & \\
\hline & 2 & & \\
\hline & 3 & & \\
\hline & 4 & & \\
\hline & 5 & & \\
\hline \multirow{5}{*}{$\begin{array}{l}\text { Other } \\
\text { List any other miscella- } \\
\text { neous keywords that } \\
\text { describe this protocol. }\end{array}$} & 1 & & \\
\hline & 2 & & \\
\hline & 3 & & \\
\hline & 4 & & \\
\hline & 5 & & \\
\hline
\end{tabular}

\section{Acknowledgments}

We thank the team of Dr Yves Méchulam (Ecole polytechnique, Palaiseau, France) for providing us with the biological samples used for illustrating this article, to the ANR (ANR-06PCVI-0018-01, ANR-11-BSV8-010-04), the CNRS and the CSIC (PICS 2011 "4DTEMmol") for supporting our research, and to GENCI-CINES/IDRIS for using HPC resources (2010x2010072174, 2011-x2010072174, 2012-x2011072174).

\section{References}

Source article(s) used to create this protocol

Jonic, S., Sorzano, C. O., \& Boisset, N. (2008). Comparison of single-particle analysis and electron tomography approaches: an overview. Journal of Microscopy, 232, 562-579.

Jonic, S., \& Venien-Bryan, C. (2009). Protein structure determination by electron cryomicroscopy. Current Opinion in Pharmacology, 9, 636-642.

Sorzano, C. O., Jonic, S., Cottevieille, M., Larquet, E., Boisset, N., \& Marco, S. (2007). 3D electron microscopy of biological nanomachines: principles and applications. European Biophysics Journal, 36, 995-1013.

Sorzano, C. O. S., de la Rosa Trevín, J. M., Otón, J., Vega, J. J., Cuenca, J., Zaldívar-Peraza, A., et al. Semiautomatic, high-throughput, high-resolution protocol for three-dimensional reconstruction of single particles in electron microscopy. In S. Alioscka \& K. Michael (Eds.), Nanoimaging: Methods and protocols. Humana Press, (in press).

\section{Referenced literature}

Connell, S. R., Takemoto, C., Wilson, D. N., Wang, H., Murayama, K., Terada, T., et al. (2007). Structural basis for interaction of the ribosome with the switch regions of GTP-bound elongation factors. Molecular Cell, 25, 751-764. 
Cottevieille, M., Larquet, E., Jonic, S., Petoukhov, M. V., Caprini, G., Paravisi, S., et al. (2008). The subnanometer resolution structure of the glutamate synthase 1.2-MDa hexamer by cryoelectron microscopy and its oligomerization behavior in solution: functional implications. Journal of Biological Chemistry, 283, 8237-8249.

Jonic, S., Sorzano, C. O., Thevenaz, P., El-Bez, C., De Carlo, S., \& Unser, M. (2005). Splinebased image-to-volume registration for three-dimensional electron microscopy. Ultramicroscopy, 103, 303-317.

Jonic, S., Sorzano, C. O., Cottevieille, M., Larquet, E., \& Boisset, N. (2007). A novel method for improvement of visualization of power spectra for sorting cryo-electron micrographs and their local areas. Journal of Structural Biology, 157, 156-167.

Leschziner, A. E., \& Nogales, E. (2007). Visualizing flexibility at molecular resolution: analysis of heterogeneity in single-particle electron microscopy reconstructions. Annual Review of Biophysics and Biomolecular Structure, 36, 43-62.

Ludtke, S. J., Baker, M. L., Chen, D. H., Song, J. L., Chuang, D. T., \& Chiu, W. (2008). De novo backbone trace of GroEL from single particle electron cryomicroscopy. Structure, 16, 441-448.

Penczek, P. A., Zhu, J., \& Frank, J. (1996). A common-lines based method for determining orientations for $N>3$ particle projections simultaneously. Ultramicroscopy, 63, 205-218.

Penczek, P. A., Zhu, J., Schröder, R., \& Frank, J. (1997). Three dimensional reconstruction with contrast transfer compensation from defocus series. Scanning Microscopy, 11, 147-154.

Radermacher, M. (1988). Three-dimensional reconstruction of single particles from random and nonrandom tilt series. Journal of Electron Microscopy Technique, 9, 359-394.

Scheres, S. H., Nunez-Ramirez, R., Sorzano, C. O., Carazo, J. M., \& Marabini, R. (2008). Image processing for electron microscopy single-particle analysis using XMIPP. Nature Protocols, 3, 977-990.

Shaikh, T. R., Gao, H., Baxter, W. T., Asturias, F. J., Boisset, N., Leith, A., \& Frank, J. (2008). SPIDER image processing for single-particle reconstruction of biological macromolecules from electron micrographs. Nature Protocols, 3, 1941-1974.

Sorzano, C. O., Jonic, S., El-Bez, C., Carazo, J. M., De Carlo, S., Thevenaz, P., et al. (2004). A multiresolution approach to orientation assignment in 3D electron microscopy of single particles. Journal of Structural Biology, 146, 381-392.

Sorzano, C. O., Jonic, S., Nunez-Ramirez, R., Boisset, N., \& Carazo, J. M. (2007). Fast, robust, and accurate determination of transmission electron microscopy contrast transfer function. Journal of Structural Biology, 160, 249-262.

Sorzano, C. O., Bilbao-Castro, J. R., Shkolnisky, Y., Alcorlo, M., Melero, R., CaffarenaFernandez, G., et al. (2010). A clustering approach to multireference alignment of singleparticle projections in electron microscopy. Journal of Structural Biology, 171, 197-206.

Venien-Bryan, C., Jonic, S., Skamnaki, V., Brown, N., Bischler, N., Oikonomakos, N. G., et al. (2009). The structure of phosphorylase kinase holoenzyme at $9.9 \AA$ resolution and location of the catalytic subunit and the substrate glycogen phosphorylase. Structure, 17, 117-127.

Zhang, X., Settembre, E., Xu, C., Dormitzer, P. R., Bellamy, R., Harrison, S. C., et al. (2008). Near-atomic resolution using electron cryomicroscopy and single-particle reconstruction. Proceedings of the National Academy of Sciences of the United States of America, 105, 1867-1872.

\section{Related literature}

Frank, J. (2006). Three-dimensional electron microscopy of macromolecular assemblies: Visualization of biological molecules in their native state. New York: Oxford University Press. 